\title{
MAX-MIN FAIR RATE CONTROL BASED ON A SADDLE-POINT CHARACTERIZATION OF SOME PERRON ROOTS
}

\author{
Stawomir Stańczak ${ }^{\star \dagger}$, MichatKaliszan ${ }^{\dagger}$ and Mario Goldenbaum ${ }^{\star}$ \\ * Heinrich-Hertz-Lehrstuhl für Informationstheorie und theoretische Informationstechnik, \\ Technische Universität Berlin, Einsteinufer 27, 10587 Berlin, Germany \\ $\dagger$ Fraunhofer Heinrich Hertz Institute, \\ Einsteinufer 37, 10587 Berlin, Germany
}

\begin{abstract}
We consider a power-controlled wireless network with an established network topology in which the communication links (transmitter-receiver pairs) are subject to general constraints on transmit powers and corrupted by the co-channel interference and background noise. In this paper, we characterize the max-min SIR power allocation and provide a saddle point characterization of this power allocation under weaker conditions. This characterization is a basis for novel algorithms for computing a max-min SIR power allocation.
\end{abstract}

Index Terms - Power Control, Max-Min Fairness, Utility Maximization

\section{INTRODUCTION}

One of the most common ideas of fairness is max-min fairness [2]. The idea behind the max-min fair approach is to treat all users as fairly as possible by making all rates as large as possible. More precisely, among all rate allocation strategies saturating a network, the max-min fair rate allocation makes the rates as equal as possible so that it is not possible to increase any rate without deteriorating other rates that are smaller or equal.

In this paper, we consider a power-controlled wireless network in which power control is the only mechanism for resource allocation. ${ }^{1}$ Consequently, max-min fairness is a power control problem and the max-min fair rate allocation is achieved by the so-called max-min fair power allocation (see also Definition 2). This power allocation is usually obtained by solving the max-min SIR problem, which is a widely studied resource allocation problem for wireless networks (see, for instance, [10], [9], [7]) and references therein). The main challenge is to solve the max-min SIR problem in a distributed manner. Another question is under which conditions the max-min SIR power allocation is equivalent to the max-min fair power allocation, and therefore achieves max-min fairness in the sense of [2].

This paper characterizes the max-min SIR power allocation for a large class of wireless networks that are not necessarily entirely coupled by interference, which was assumed in [6]. For this class of wireless networks (specified by Condition (A.4)), it is shown that the max-min SIR power allocation is unique and equal to the max-min

The work was supported by the German Research Foundation (DFG) under grant STA864/3-1 and by the German Federal Ministry of Education and Research (BMBF) under grant 01BU920.

${ }^{1}$ There is no scheduling and all users (transmitter-receiver pairs) share a common bandwidth fair power allocation (which is always unique). Furthermore, we use a class of utility functions to prove a saddle-point characterization of the max-min SIR power allocation. For brevity, the characterization is proven for networks that are entirely coupled by interference but the result can be easily extended to capture the more general case. However, in contrast to the previous work [6,8], the utility functions are not necessarily differentiable functions. We finish the paper by presenting a saddle-point algorithm that converges to the max-min SIR power allocation. A simulation result illustrates the convergence behavior of the algorithm.

\section{DEFINITIONS AND PROBLEM STATEMENT}

We consider a wireless network with an established network topology, in which $K \geq 2$ users (point-to-point logical links) share a common wireless spectrum and transmit their independent data concurrently. Let $\mathcal{K}=\{1, \ldots, K\}$ and let $\mathbf{p}=\left(p_{1}, \ldots, p_{K}\right) \geq 0$, be the power vector or power allocation, where $p_{k}, k \in \mathcal{K}$, is the transmit power of user $k$. Due to power constraints, we have $\mathbf{p} \in \mathrm{P}$ where $\mathrm{P}$ is a compact convex set of all feasible power allocations (called feasible power region) assumed to $\mathrm{be}^{2}$

$$
\mathrm{P}=\left\{\mathbf{p} \in \mathbb{R}_{+}^{K}: \mathbf{C p} \leq \hat{\mathbf{p}}, \mathbf{C} \in\{0,1\}^{N \times K}\right\} \subset \mathbb{R}^{K}
$$

for some given $\hat{\mathbf{p}}=\left(P_{1}, \ldots, P_{N}\right)>0, N \geq 1$ (the number of power constraints) and $\mathbf{C}$ with at least one 1 in each column. Let $\mathcal{N}=\{1, \ldots, N\}$. The main figure of merit is the SIR at the output of each receiver given by

(A.1) $\operatorname{SIR}_{k}(\mathbf{p})=p_{k} / I_{k}(\mathbf{p}), k \in \mathcal{K}$, where the interference function $I_{k}$ is $I_{k}(\mathbf{p})=(\mathbf{V p}+\mathbf{z})_{k}=\sum_{l=1}^{K} v_{k, l} p_{l}+z_{k}$.

$\mathbf{V}:=\left(v_{k, l}\right) \in \mathbb{R}_{+}^{K \times K}$ is the gain matrix, $v_{k, l}=V_{k, l} / V_{k, k}$ if $l \neq k$ and 0 if $l=k$ where $V_{k, l} \geq 0$ with $V_{k, k}>0$ is the attenuation of the power from transmitter $l$ to receiver $k$. The $k$ th entry of $\mathbf{z}:=$ $\left(z_{1}, \ldots, z_{K}\right)$ is $z_{k}=\sigma_{k}^{2} / V_{k, k}$, where $\sigma_{k}^{2}>0$ is the noise variance at the receiver output.

Definition 1 (Max-Min SIR power vector). The power vector $\overline{\mathbf{p}}$ is said to be a max-min SIR power vector/allocation if

$$
\min _{k \in \mathcal{K}} \operatorname{SIR}_{k}(\overline{\mathbf{p}})=\sup _{\mathbf{p} \in \mathrm{P}} \min _{k \in \mathcal{K}} \operatorname{SIR}_{k}(\mathbf{p}) .
$$

where the supremum is attained because $\min _{k \in \mathcal{K}} \operatorname{SIR}_{k}(\mathbf{p})$ is continuous on the compact set $\mathrm{P}$.

\footnotetext{
${ }^{2} \mathbb{R}_{+}, \mathbb{R}_{++}$are nonnegative and positive reals, respectively.
} 
Since $\overline{\mathbf{p}}>0$, we can focus on $\mathrm{P}_{+}:=\mathrm{P} \cap \mathbb{R}_{++}^{K}$. Considering this and (1), $\overline{\mathbf{p}}$ defined by (2) can be written as

$$
\overline{\mathbf{p}}=\underset{\mathbf{p}>0}{\arg \max } \min _{k \in \mathcal{K}}\left(\operatorname{SIR}_{k}(\mathbf{p})\right) \quad \text { s.t. } \quad \max _{n \in \mathcal{N}} g_{n}(\mathbf{p}) \leq 1
$$

where

$$
g_{n}(\mathbf{p}):=1 / P_{n} \mathbf{c}_{n}^{T} \mathbf{p} \leq 1, \quad n \in \mathcal{N}
$$

and $\mathbf{c}_{n} \in\{0,1\}^{K}$ is equal to the $n$th row of $\mathbf{C}$. It is pointed out that there may be multiple max-min SIR power allocations (which stands in contrast to the max-min fair power allocation). Let $\overline{\mathrm{P}} \subset \mathrm{P}$ be the set of all max-min SIR power allocations. Later we will prove a sufficient condition for $|\overline{\mathrm{P}}|=1$.

Now let $\phi: \mathbb{R}_{++} \rightarrow \mathrm{Q} \subseteq \mathbb{R}$ be

(A.2) any continuous and strictly increasing utility function.

We assume that

(A.3) $\phi\left(e^{x}\right), x \in \mathbb{R}$, is a concave function.

Examples of functions satisfying (A.2) and (A.3) are $x \mapsto \log (x), x>$ 0 , and $x \mapsto-1 / x^{n}, n \geq 1, x>0$. By strict increasingness, $\phi\left(\min _{k \in \mathcal{K}} \operatorname{SIR}_{k}(\mathbf{p})\right)=\min _{k \in \mathcal{K}} \phi\left(\operatorname{SIR}_{k}(\mathbf{p})\right)$ for all $\mathbf{p}>0$. Thus, as $\overline{\mathbf{p}} \in \mathrm{P}_{+}$, we have

$$
\overline{\mathbf{p}}=\underset{\mathbf{p} \in \mathrm{P}_{+}}{\arg \max } \min _{k \in \mathcal{K}} \phi\left(\operatorname{SIR}_{k}(\mathbf{p})\right) .
$$

Given $\phi$, let $\mathrm{F} \subset \mathrm{Q}^{K}$ be the feasible QoS region:

$$
\mathrm{F}=\left\{\boldsymbol{\eta} \in \mathrm{Q}^{K}: \eta_{k} \leq \phi\left(\operatorname{SIR}_{k}(\mathbf{p})\right), k \in \mathcal{K}, \mathbf{p} \in \mathrm{P}_{+}\right\} .
$$

With the above, the following can be said about F.

Observation 1. There is a bijective continuous map from $\mathrm{F}$ onto $\mathrm{P}_{+}$. If (A.2)-(A.3) hold, then $\mathrm{F}$ is downward comprehensive and convex. Finally, $\overline{\mathbf{p}}$ achieves a point on the boundary of $\mathrm{F}$.

Note that the boundary of $\mathrm{F}$ is the set of all points of $\mathrm{F}$ such that, if $\mathbf{p}$ is the corresponding power vector in (6), then $\mathbf{C p} \leq \hat{\mathbf{p}}$ holds with at least one equality.

\section{CHARACTERIZATION OF MAX-MIN SIR POWER ALLOCATION}

In this section we recall some of previous results that are used later in this paper $[6,7,8]$. We can easily observe the following.

Lemma 1. The following holds $(i) \max _{n \in \mathcal{N}} g_{n}(\mathbf{p})=1$ for any $\mathbf{p} \in \overline{\mathrm{P}}$ and (ii) there exists a unique $\mathbf{p} \in \overline{\mathrm{P}}$ such that

$$
\forall \forall_{k \in \mathcal{K}} 1 / \operatorname{SIR}_{k}(\mathbf{p})=\beta, \beta>0 .
$$

Let us have a closer look at the max-min SIR power allocation satisfying (7). An immediate consequence of (ii) with (i) in Lemma 1 is that there is a unique $\overline{\mathbf{p}} \in \overline{\mathrm{P}}$ and some $n \in \mathcal{N}$ such that

$$
\beta \overline{\mathbf{p}}=\mathbf{V} \overline{\mathbf{p}}+\mathbf{z} \quad g_{n}(\overline{\mathbf{p}})=1 .
$$

From this we have $\beta \overline{\mathbf{p}}=\mathbf{V} \overline{\mathbf{p}}+\mathbf{z} \cdot g_{n}(\overline{\mathbf{p}})$ for each $n \in \mathcal{N}_{0}(\overline{\mathbf{p}})$ where $\mathcal{N}_{0}(\mathbf{p}):=\left\{m \in \mathcal{N}: m=\arg \max _{n \in \mathcal{N}} g_{n}(\mathbf{p})\right\}$. Note that by $(i)$ of Lemma 1, the cardinality of $\mathcal{N}_{0}(\overline{\mathbf{p}})$ must be larger than or equal to 1 . Consequently, (8) is equivalent to

$$
\beta \overline{\mathbf{p}}=\mathbf{B}^{(n)} \overline{\mathbf{p}}, \quad \beta>0, \overline{\mathbf{p}} \in \mathbb{R}_{++}^{K+1}, n \in \mathcal{N}_{0}(\overline{\mathbf{p}}) .
$$

with $\mathbf{B}^{(n)} \in \mathbb{R}_{+}^{K \times K}$ (for each $n \in \mathcal{N}$ ) defined to be

$$
\mathbf{B}^{(n)}:=\mathbf{V}+\frac{1}{P_{n}} \mathbf{z c}_{n}^{T}=\left(\mathbf{V}+\frac{1}{P_{n}} \mathbf{z c}_{n}^{T}\right) .
$$

Unless otherwise stated, it is assumed that
(A.4) $\mathbf{B}^{(n)} \geq 0$ is irreducible for some $n \in \mathcal{N}_{0}(\overline{\mathbf{p}})$.

Notice that the above assumption is weaker than irreducibility of $\mathbf{V}$, in which case the network is entirely coupled by interference. Indeed, if $\mathbf{V}$ is irreducible, then $\mathbf{B}^{(n)}$ is irreducible for each $n \in \mathcal{N}$, no matter how $\mathbf{c}_{n}$ is chosen. On the other hand, if $\mathbf{c}_{n}$ is positive for some $n$, then $\mathbf{B}^{(n)}$ is irreducible regardless of the choice of $\mathbf{V} \geq 0$. This is the case when users are subject to a sum power constraint, and therefore are entirely coupled by the power constraints.

Theorem 1. If (A.4) holds, then $\overline{\mathbf{p}} \in \mathrm{P}_{+}$solves the max-min SIRbalancing problem (3) if and only if there is $n \in \mathcal{N}_{0}(\overline{\mathbf{p}})$ such that $\overline{\mathbf{p}}$ is a unique positive right eigenvector of $\mathbf{B}^{(n)}$ associated with $\beta=$ $\rho\left(\mathbf{B}^{(n)}\right)>0$ such that $g_{n}(\overline{\mathbf{p}})=1$.

Theorem 1 implies that if (A.4) is true, then $\overline{\mathbf{p}}>0$ is the (positive) right eigenvector of $\mathbf{B}^{(n)}$ associated with $\rho\left(\mathbf{B}^{(n)}\right) \in \sigma\left(\mathbf{B}^{(n)}\right)$ for some $n \in \mathcal{N}_{0}(\overline{\mathbf{p}})$ where $\sigma\left(\mathbf{B}^{(n)}\right)$ is used to denote the spectrum of the matrix $\mathbf{B}^{(n)}$. Another consequence of the theorem is the following corollary.

Corollary 1. Suppose that (A.4) holds. Then, $\overline{\mathbf{p}}$ is the unique maxmin fair power vector.

If the rate is a strictly increasing function of the SIR, max-min fairness can be defined as follows.

Definition 2 (Max-Min Fair Power Allocation). $\hat{\mathbf{p}} \in \mathrm{P}$ is said to be max-min fair power allocation if any $\operatorname{SIR}_{k}(\hat{\mathbf{p}})$ cannot be increased without decreasing some $\operatorname{SIR}_{l}(\hat{\mathbf{p}}), l \neq k$, which is smaller than or equal to $\operatorname{SIR}_{k}(\hat{\mathbf{p}})$.

The problem is, however, that $\mathcal{N}_{0}(\overline{\mathbf{p}})$ is not known as this set is determined by the solution to the max-min SIR-balancing problem, and hence its determination is itself a part of the problem. As the SIR targets are feasible if and only if they are met under $\overline{\mathbf{p}}$, the following characterization of the set $\mathcal{N}_{0}(\overline{\mathbf{p}})$ immediately follows from [4].

Theorem 2 ([4]). We have

$$
\mathcal{N}_{0}:=\mathcal{N}_{0}(\overline{\mathbf{p}})=\left\{n_{0} \in \mathcal{N}: n_{0}=\underset{n \in \mathcal{N}}{\arg \max } \rho\left(\mathbf{B}^{(n)}\right)\right\} .
$$

Moreover, $\boldsymbol{\eta} \in \mathrm{F}$ if and only if $\max _{n \in \mathcal{N}} \rho\left(\operatorname{diag}(\boldsymbol{\eta}) \mathbf{B}^{(n)}\right) \leq 1$ where $\operatorname{diag}(\boldsymbol{\eta})=\operatorname{diag}\left(\eta_{1}, \ldots, \eta_{K}\right)$.

It is worth pointing out that by Theorem 1 , the uniqueness of the max-min SIR power allocation is ensured if $\mathbf{V}$ is irreducible [3, 5] since, as mentioned before, the irreducibility property implies (A.4). The difference is that if the gain matrix is irreducible, then $\overline{\mathbf{p}}$ is the normalized positive right eigenvector of $\mathbf{B}^{(n)}$ for each $n \in \mathcal{N}_{0}(\overline{\mathbf{p}})$, whereas under (A.4) this is ensured only for some $n \in \mathcal{N}_{0}(\overline{\mathbf{p}})$. For brevity, in what follows, we assume that

(A.5) $\mathrm{V} \geq 0$ is irreducible.

It is however emphasized that the results can be extended to the weaker condition (A.4).

\section{SADDLE POINT CHARACTERIZATION}

Let $\Pi_{K}:=\left\{\mathbf{x} \in \mathbb{R}_{+}^{K}:\|\mathbf{x}\|_{1}=1\right\}$ and $\Pi_{K}^{+}=\Pi_{K} \cap \mathbb{R}_{++}^{K}$. We define $G: \Pi_{K}^{+} \times \mathrm{P}_{+} \rightarrow \mathbb{R}$ as

$$
G(\mathbf{u}, \mathbf{p}):=-\sum_{k \in \mathcal{K}} u_{k} \phi\left(\operatorname{SIR}_{k}(\mathbf{p})\right)=\sum_{k \in \mathcal{K}} u_{k} \theta\left(\frac{1}{\operatorname{SIR}_{k}(\mathbf{p})}\right)
$$


where

$$
\theta(x):=-\phi(1 / x), \quad x>0
$$

is a strictly increasing function. Thus, any power vector minimizing $G(\mathbf{w}, \mathbf{p})$ with respect to $\mathbf{p} \in \mathrm{P}$ for some given weight vector $\mathbf{w}>0$ is optimal in the sense of the utility maximization problem considered in [7]. The following theorem, which is used later for the saddle-point characterization, extends previous results [7] to a class of continuous (but not necessarily differentiable) functions.

Theorem 3. Assume that (A.1)-(A.5) hold. Let $\mathbf{B}=\mathbf{B}^{(n)}$ for some $n \in \mathcal{N}$, and let $\mathbf{w}=\mathbf{y} \circ \mathbf{x} \in \Pi_{K}^{+} \subset \Pi_{K}$ where $\mathbf{y}$ and $\mathbf{x}$ are positive left and right eigenvectors of $\mathbf{B}$. Then, for all $\mathbf{p}>0$,

$$
\theta(\rho(\mathbf{B})) \leq \sum_{k \in \mathcal{K}} w_{k} \theta\left(\frac{(\mathbf{B p})_{k}}{p_{k}}\right) .
$$

Equality holds if and only if $\mathbf{p}=\mathbf{x}>0$.

Proof. As B is irreducible, it follows from [7] that

$$
\log \frac{1}{\rho(\mathbf{B})} \geq \sum_{k \in \mathcal{K}} w_{k} \log \frac{p_{k}}{(\mathbf{B p})_{k}}
$$

for all $\mathbf{p}>0$ with equality if and only if $\mathbf{p}=\mathbf{x}$. Let $\varphi(x)=$ $-\phi(x), x>0$. By (A.2), $\varphi\left(e^{x}\right), x \in \mathbb{R}$, is strictly decreasing. So, taking the function $\varphi\left(e^{x}\right)$ of both sides of (14) yields

$$
\varphi\left(e^{\log \frac{1}{\rho(\mathbf{B})}}\right)=\varphi\left(\frac{1}{\rho(\mathbf{B})}\right) \leq \varphi\left(e^{\sum_{k \in \mathcal{K}} w_{k} \log \frac{p_{k}}{(\mathbf{B} \mathbf{p})_{k}}}\right)
$$

for all $\mathbf{p}>0$. As $\mathbb{R} \rightarrow \mathrm{Q}: x \mapsto \varphi\left(e^{x}\right)$ is strictly decreasing, we have equality if and only if $\mathbf{p}=\mathbf{x}$. By (A.3), $\varphi_{e}(x)=\varphi\left(e^{x}\right)$ is convex. Thus, since $\|\mathbf{w}\|_{1}=1$, applying Jensen's inequality gives

$$
\begin{aligned}
\varphi\left(\frac{1}{\rho(\mathbf{B})}\right) & \leq \varphi_{e}\left(\sum_{k \in \mathcal{K}} w_{k} \log \frac{p_{k}}{(\mathbf{B p})_{k}}\right) \leq \sum_{k \in \mathcal{K}} w_{k} \varphi_{e}\left(\log \frac{p_{k}}{(\mathbf{B p})_{k}}\right) \\
& =\sum_{k \in \mathcal{K}} w_{k} \varphi\left(e^{\log \frac{p_{k}}{(\mathbf{B} \mathbf{p})_{k}}}\right)=\sum_{k \in \mathcal{K}} w_{k} \varphi\left(\frac{p_{k}}{(\mathbf{B p})_{k}}\right)
\end{aligned}
$$

for all $\mathbf{p}>0$. Equality if and only if $\mathbf{p}=\mathbf{x}$. Now defining $\theta(x)=$ $\varphi(1 / x), x>0$, proves the theorem.

Corollary 2. Let the conditions and definitions be as in Theorem 3. Then,

$$
\theta(\rho(\mathbf{B}))=\min _{\mathbf{p}>0} \max _{\mathbf{u} \in \Pi_{K}} \sum_{k \in \mathcal{K}} u_{k} \theta\left(\frac{(\mathbf{B} \mathbf{p})_{k}}{p_{k}}\right)
$$

with the minimum attained if and only if $\mathbf{p}=\mathbf{x}>0$.

Proof. Clearly, for any $\mathbf{u} \in \Pi_{K}$ and all $\mathbf{p}>0$, one has

$$
\begin{aligned}
& \inf _{\mathbf{p}>0} \sum_{k \in \mathcal{K}} u_{k} \theta\left(\frac{(\mathbf{B p})_{k}}{p_{k}}\right) \leq \inf _{\mathbf{p}>0} \max _{k \in \mathcal{K}} \theta\left(\frac{(\mathbf{B p})_{k}}{p_{k}}\right) \\
& =\theta\left(\inf _{\mathbf{p}>0} \max _{k \in \mathcal{K}} \frac{(\mathbf{B p})_{k}}{p_{k}}\right) \stackrel{(a)}{=} \theta\left(\min _{\mathbf{p}>0} \max _{k \in \mathcal{K}} \frac{(\mathbf{B p})_{k}}{p_{k}}\right) \stackrel{(b)}{=} \theta(\rho(\mathbf{B}))
\end{aligned}
$$

where (a) and (b) follow from Collatz-Wielandt formula [5] as B is irreducible. Moreover, for any $\mathbf{u} \in \Pi_{K}^{+}$(positive), equality holds if and only if $\mathbf{p}=\mathbf{x}>0$. Combining this with Theorem 3 shows (15), with the minimum attained if and only if $\mathbf{p}=\mathbf{x}>0$.

Notice that by the proof of Corollary 2, (13) holds for all $\mathbf{p}>0$ if and only if $\mathbf{w}=c \mathbf{y} \circ \mathbf{x}$ for some $c>0$.

In the proof of the "min-max" part, we use the following lemma.
Lemma 2. Suppose that (A.1)-(A.5) hold and let $\mathbf{B}=\mathbf{B}^{(n)}$ for some $n \in \mathcal{N}$. Then, the function $E: \Pi_{K}^{+} \rightarrow \mathbb{R}$ given by

$$
E(\mathbf{u}):=\min _{\mathbf{p}>0} \sum_{k \in \mathcal{K}} u_{k} \theta\left(\frac{(\mathbf{B p})_{k}}{p_{k}}\right)
$$

is strictly concave.

The proof is omitted here due to the lack of space but we point out that the lemma extends [7, Lemma 1.32] to continuous but not necessarily differentiable functions

Theorem 4. Let the conditions of Theorem 3 be satisfied, and let $\mathbf{B}=\mathbf{B}^{(n)}$ for an arbitrary $n \in \mathcal{N}$. Then,

$$
\max _{\mathbf{u} \in \Pi_{K}} \min _{\mathbf{p}>0} \sum_{k \in \mathcal{K}} u_{k} \theta\left(\frac{(\mathbf{B} \mathbf{p})_{k}}{p_{k}}\right)=\theta(\rho(\mathbf{B}))
$$

The minimum is attained and $\mathbf{u} \in \Pi_{K}$ is a minimizer if and only if $\mathbf{u}=\mathbf{w}$ where $\mathbf{w}=\mathbf{y} \circ \mathbf{x}$.

Sketch of the proof. Proceeding as in the proof of Corollary 2 shows that, for any $\mathbf{u} \in \Pi_{K}$, one has $E(\mathbf{u}) \leq \theta(\rho(\mathbf{B}))$. A careful examination of this bound and Theorem 3 leads us to conclude that $\mathbf{w}=\mathbf{y} \circ \mathbf{x}$ is a maximizer of $E$ over $\Pi_{K}$. Due to strict concavity of $E$ (Lemma 2), the maximizer is unique, and the theorem follows.

Now combining Corollary 2 and Theorem 4 yields the saddle point characterization of the Perron root. Note that the theorem extends the results of $[6,7]$ (see also references therein) to nondifferentiable functions.

Theorem 5. Suppose that (A.2) and (A.3) hold, and $\mathbf{V}$ is irreducible. Let $\mathbf{w}=\mathbf{y} \circ \mathbf{x}>0$ where $\rho\left(\mathbf{B}^{\left(n_{0}\right)}\right) \mathbf{x}=\mathbf{B}^{\left(n_{0}\right)} \mathbf{x}, \rho\left(\mathbf{B}^{\left(n_{0}\right)}\right) \mathbf{y}=$ $\left(\mathbf{B}^{\left(n_{0}\right)}\right)^{T} \mathbf{y}$ with $\mathbf{y}^{T} \mathbf{x}=1$. Then, for each $n_{0} \in \mathcal{N}_{0}$,

$$
\theta\left(\rho\left(\mathbf{B}^{\left(n_{0}\right)}\right)\right)=\max _{\mathbf{u} \in \Pi_{K}} \min _{\mathbf{p} \in \mathrm{P}} G(\mathbf{u}, \mathbf{p})=\min _{\mathbf{p} \in \mathrm{P}} \max _{\mathbf{u} \in \Pi_{K}} G(\mathbf{u}, \mathbf{p})
$$

and $(\mathbf{w}, \overline{\mathbf{p}})$ is the unique saddle point in $\Pi_{K} \times \mathrm{P}_{+}$.

\section{ALGORITHM AND SIMULATIONS}

Now the goal is to use the characterization of Theorem 5 to design an iterative saddle-point algorithm that maximizes $G(\mathbf{u}, \mathbf{p})$ with respect to $\mathbf{u} \in \Pi_{K}$ and simultaneously minimizes this function over the feasible power region P. Due to Theorem 5, the algorithm will converge to a saddle point $\left(\mathbf{u}^{*}, \mathbf{p}^{*}\right)$ of $G(\mathbf{u}, \mathbf{p})$, which is a unique point in $\Pi_{K} \times \mathrm{P}$. For brevity, we assume in this section that $\phi$ is continuously differentiable and point out that the algorithms can be extended to incorporate non-differentiable functions by considering sub-gradient methods [1].

The function $G(\mathbf{u}, \mathbf{p})$ is in general not concave in $\mathbf{p} \in \mathrm{P}$ but by Theorem 5, we have $\mathbf{p}^{*}>0$ (and $\mathbf{u}^{*}>0$ ). Therefore, by [7, Section 6.2], $G_{s}(\mathbf{u}, \mathbf{s}):=G\left(\mathbf{u}, e^{\mathbf{s}}\right)$ is well-defined on $\Pi_{K} \times \mathrm{S}$ with $\mathrm{S}:=\left\{\mathbf{s} \in \mathbb{R}^{K}: \mathbf{s}=\log (\mathbf{p}), \mathbf{p} \in \mathrm{P}_{+}\right\}$and concave with respect to $\mathbf{s} \in \mathrm{S}$. So, $G_{s}(\mathbf{u}, \mathbf{s})$ is a convex-concave function on $\Pi_{K} \times \mathrm{S}$.

A straightforward approach consists in applying the gradient projection method, in which case the algorithm takes the form:

$$
\begin{aligned}
& \mathbf{u}(n+1)=P_{\Pi_{K}}\left[\mathbf{u}(n)+\delta \nabla_{\mathbf{u}} G\left(\mathbf{u}(n), e^{\mathbf{s}(n)}\right)\right] \\
& \mathbf{s}(n+1)=P_{\mathrm{S}}\left[\mathbf{s}(n)-\delta \nabla_{\mathbf{s}} G\left(\mathbf{u}(n), e^{\mathbf{s}(n)}\right)\right]
\end{aligned}
$$


where $\delta>0$ is a suitably chosen step size, and $P_{\Pi_{K}}[\mathbf{x}]=$ $\arg \min _{\mathbf{u} \in \Pi_{K}}\|\mathbf{x}-\mathbf{u}\|_{2}$ and $P_{\mathrm{S}}[\mathbf{y}]=\arg \min _{\mathbf{s} \in \mathrm{S}}\|\mathbf{y}-\mathbf{s}\|_{2}$ denote the projections of $\mathbf{x} \in \mathbb{R}^{K}$ on $\Pi_{K}$ and $\mathbf{y} \in \mathbb{R}^{K}$ on $\mathrm{S}$, respectively. The gradients in (19) can be easily computed to yield $\left(\nabla_{\mathbf{u}} G\left(\mathbf{u}, e^{\mathbf{s}}\right)\right)_{k}=f_{k}(\mathbf{s}), k \in \mathcal{K}$ and $\left(\nabla_{\mathbf{s}} G\left(\mathbf{u}, e^{\mathbf{s}}\right)\right)_{k}=$ $e^{s_{k}} \sum_{l \neq k} v_{l, k} \frac{g_{l}\left(e^{\mathbf{s}}\right)}{I_{l}\left(e^{\mathbf{s}}\right)}-g_{k}\left(e^{\mathbf{s}}\right), k \in \mathcal{K}$ where $f_{k}$ and $g_{k}$ are

$$
\begin{aligned}
& f_{k}(\mathbf{s}):=\theta\left(1 / \operatorname{SIR}_{k}\left(e^{\mathbf{s}}\right)\right), k \in \mathcal{K} \\
& g_{k}\left(e^{\mathbf{s}}\right):=\frac{u_{k} \theta^{\prime}\left(1 / \operatorname{SIR}_{k}\left(e^{\mathbf{s}}\right)\right)}{\operatorname{SIR}_{k}\left(e^{\mathbf{s}}\right)}, k \in \mathcal{K} .
\end{aligned}
$$

with $I_{k}$ and $\phi$ given by (A.1) and (12), respectively. In general, the projection on S may be difficult to implement in a distributed manner but still there are many cases of practical relevance, in which the projection is amenable to distributed implementation. For instance, under individual power constraints, we have $\mathrm{P}=\left\{\mathbf{p} \in \mathbb{R}_{+}^{K}\right.$ : $\left.\forall_{k \in \mathcal{K}} p_{k} \leq \hat{p}_{k}\right\}$ so that the projection $P_{\mathrm{S}}[\mathbf{y}]$ reduces to $\left(P_{\mathrm{S}}[\mathbf{y}]\right)_{k}=$ $\min \left(y_{k}, \log \left(\hat{p}_{k}\right)\right), k \in \mathcal{K}$. The projection can also be performed locally in the case of per-node power constraints.

To facilitate distributed implementation, we replace the equality constraint $\|\mathbf{u}\|_{1}=1$ with two inequalities, $\|\mathbf{u}\|_{1}-1 \geq 0$ and $1-$ $\|\mathbf{u}\|_{1} \geq 0 .{ }^{3}$ Augmenting the constraints yields a Lagrange function $L: \mathbb{R}^{\bar{K}} \times \mathrm{S} \times \mathbb{R}_{+}^{2} \rightarrow \mathbb{R}$ given by

$$
L(\mathbf{u}, \mathbf{s}, \boldsymbol{\lambda})=G\left(\mathbf{u}, e^{\mathbf{s}}\right)+\sum_{j=1}^{2} \lambda_{j}(-1)^{j}\left(1-\sum_{k \in \mathcal{K}} u_{k}\right) .
$$

Due to the convex-concave property, there is no dual gap and the saddle point of $G(\mathbf{u}, \mathbf{p})$ can be found by maximizing the Lagrange function over $\Pi_{K}$ and simultaneously minimizing it with respect to $(\mathbf{s}, \boldsymbol{\lambda}) \in \mathrm{S} \times \mathbb{R}_{+}^{2}$. The algorithm is formally stated as follows:

$$
\begin{aligned}
\mathbf{u}(n+1) & =\max \left[\mathbf{u}(n)+\delta \nabla_{\mathbf{u}} L(\mathbf{u}(n), \mathbf{s}(n), \boldsymbol{\lambda}(n)), 0\right] \\
\mathbf{s}(n+1) & =P_{\mathbf{S}}\left[\mathbf{s}(n)-\delta \nabla_{\mathbf{s}} L(\mathbf{u}(n), \mathbf{s}(n), \boldsymbol{\lambda}(n))\right] \\
\boldsymbol{\lambda}(n+1) & =\max \left[\boldsymbol{\lambda}(n)-\delta \nabla_{\boldsymbol{\lambda}} L(\mathbf{u}(n), \mathbf{s}(n), \boldsymbol{\lambda}(n)), 0\right]
\end{aligned}
$$

where $P_{\mathrm{S}}[\mathbf{y}]$ is as before. Furthermore, we have

$$
\begin{aligned}
& \left(\nabla_{\mathbf{u}} L(\mathbf{u}, \mathbf{s}, \boldsymbol{\lambda})\right)_{k}=f_{k}(\mathbf{s})+\lambda_{1}-\lambda_{2}, k \in \mathcal{K} \\
& \left(\nabla_{\mathbf{s}} L(\mathbf{u}, \mathbf{s}, \boldsymbol{\lambda})\right)_{k}=e^{s_{k}} \sum_{l \neq k} v_{l, k} \frac{g_{l}\left(e^{\mathbf{s}}\right)}{I_{l}\left(e^{\mathbf{s}}\right)}-g_{k}\left(e^{\mathbf{s}}\right), k \in \mathcal{K} \\
& \left(\nabla_{\lambda} L(\mathbf{u}, \mathbf{s}, \boldsymbol{\lambda})\right)_{j}=(-1)^{j}\left(1-\sum_{k \in \mathcal{K}} u_{k}\right), j=1,2
\end{aligned}
$$

with $f_{k}$ and $g_{k}$ are given by (20) and (21), respectively.

Notice that in certain cases one of the two inequalities $\|\mathbf{u}\|_{1}-$ $1 \geq 0$ and $1-\|\mathbf{u}\|_{1} \geq 0$ may be redundant. This is for instance true if $\forall_{x>0} \theta(x)>0$, in which case can be simplified by omitting the constraint and the corresponding multiplier $\lambda_{j}$ from the Lagrange function (22).

Compared with (19), the projection $P_{\Pi_{K}}[\mathbf{u}]$ is not needed in (23) but it is necessary for each user to know the values of $\sum_{k \in \mathcal{K}} u_{k}$ and $\boldsymbol{\lambda}$. Obtaining them can be achieved using appropriate auxiliary protocols which is in general a less complex task than computing $P_{\Pi_{K}}[\mathbf{u}]$. An efficient distributed computation of $\left(\nabla_{\mathbf{s}} L(\mathbf{u}, \mathbf{s}, \boldsymbol{\lambda})\right)_{k}$ can be realized with the help of adjoint network [7].

In what follows, we demonstrate the behavior of algorithm (23) by a simple example.

Example 1. Let a network be given consisting of $K=10$ active users, where each is equipped with a unity individual power constraint (i.e., according to (1), $\mathbf{C p} \leq \hat{\mathbf{p}}=(1, \ldots, 1)$, with $\mathbf{C}$ the

\footnotetext{
${ }^{3}$ In general it is not sufficient to replace $\|\mathbf{u}\|_{1}=1$ by $1-\|\mathbf{u}\|_{1} \geq 0$.
}

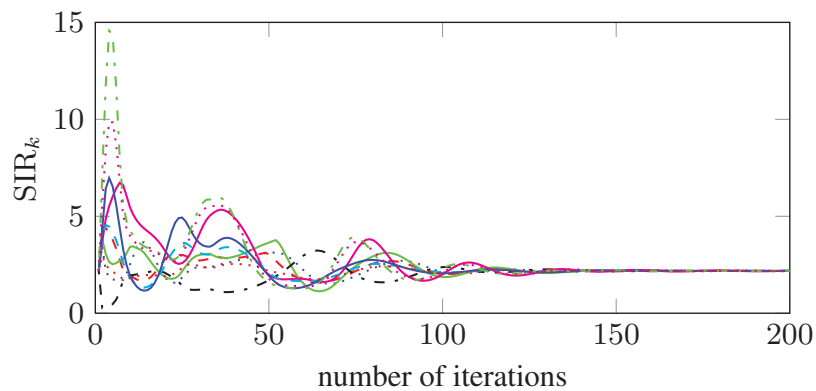

Fig. 1. Evolution of the SIR's of all users $k \in \mathcal{K}$ (i.e., $\operatorname{SIR}_{k}$ ), with $|\mathcal{K}|=10$, as a result of algorithm (23).

$K \times K$ identity matrix). The entries of the gain matrix $\mathbf{V} \in \mathbb{R}_{+}^{K \times K}$ are independent, identically and exponentially distributed ( $\mathbf{V}$ is irreducible with probability one) with expected values equal to $1 / 10$. Furthermore, $\sigma_{k}^{2}=1 / 5$, for all $k \in \mathcal{K}$.

Figure 1 shows the evolution of the $\operatorname{SIR}_{k}$ of each user $k \in \mathcal{K}$, based on the iterations (23), for an appropriately chosen step size $\delta>0$. The plots confirm Theorem 5 since all users converge to the same SIR value.

\section{REFERENCES}

[1] D.P. Bertsekas. Nonlinear Programming. Athena Scientific, Belmont, Massachusetts, 2003.

[2] D.P. Bertsekas and R.G. Gallager. Data Networks. PrenticeHall, Englewood Cliffs, 1992.

[3] R.A. Horn and C.R. Johnson. Matrix Analysis. Cambridge University Press, 1985.

[4] H. Mahdavi-Doost, M. Ebrahimi, and A. K. Khandani. Characterization of rate region in interference channels with constrained power. In Proc. IEEE International Symposium on Information Theory (ISIT), Nice, France, 2007.

[5] C. D. Meyer. Matrix Analysis and Applied Linear Algebra. SIAM, Philadelphia, 2000.

[6] S. Stanczak, M. Kaliszan, N. Bambos, and M. Wiczanowski. A characterization of max-min SIR-balanced power allocation with applications. In Proc. IEEE International Symposium on Information Theory (ISIT), Seoul, Korea, June 28-July 32009.

[7] S. Stanczak, M. Wiczanowski, and H. Boche. Fundamentals of Resource Allocation in Wireless Networks, volume 3 of Foundations in Signal Processing, Communications and Networking. Springer, Berlin, 2009.

[8] C.W. Tan, M. Chiang, and R. Srikant. Fast algorithms and performance bounds for sum rate maximization in wireless networks. In Proc. 28th IEEE Conference on Computer Communications (INFOCOM), Rio de Janeiro, Brazil, April, 19-25 2009.

[9] W. Yang and G. Xu. Optimal downlink power assignment for smart antenna systems. In Proc. IEEE International Conference on Acoustics, Speech, and Signal Processing (ICASSP), Seattle, WA, USA, May 1998.

[10] J. Zander and S.-L. Kim. Radio Resource Management for Wireless Networks. Artech House, Boston, London, 2001. 